\title{
Soll der Versicherungsanspruch mit dem Budget enden?
}

\author{
Jürg Schlup \\ Dr. med., Präsident der FMH
}

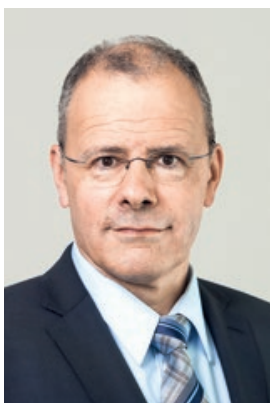

Literatu

1 Entwurf Bundesgesetz über die Krankenversicherung (KVG) (Massnahmen rung (KVG) (Massnahm Paket1).

219.046 Botschaft zur Änderung des Bundesgesetzes über die Krankenversicherung (Massnahmen zur Kostendämpfung - Paket 1) vom 21.8 .2019

Medienmitteilung des BAG, 8.3.2019. Bundesrat BAG, 8.3.2019. Bundesrat
lässt Zielvorgabe für die Kostenentwicklung im Gesundheitswesen erarbeiten.

4 Oliver Hach. Wenn Politik und Verwaltung «Mengen regulieren», Budget überschritten: Ärztin soll 300000 Euro zahlen. Schweiz Ärzteztg. 2020;101(7):196-7.

Busse, Reinhard; Blankart, Rudolf (2017). KostendämpRudolf(2017). Kostendäm
fungsmassnahmen zur Entlastung der obligatorischen Krankenpflegeversicherung (OKP). Erfahrungen aus Deutschland. Studie zu Handen der Studiezu Handen der Ex pertengruppe Kostendämpfung. Berlin und Bern: Technische Universität Berlin und Universität Bern (unveröffentlicht).

Der Bund, 21.12.2019, Artikel «Berset leitet eine Artike Bersetleiteteine Bund legt 7 hl der IVRenten fest» (S.1 und 9).

7 NZZ, 13.1.2020, Fabian Schäfer, Misstraut Berset seinen eigenen Leuten? Der Sozialminister lässt angebliche Sparvorgaben in de Invalidenversicherung

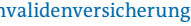
untersuchen.
Das Jahr 2020 gilt nach dem chinesischen Tierkreismodell als Jahr des Neubeginns und der Erneuerung. Auch die Schweizer Gesundheitspolitik verspricht mit ihren Gesetzesvorhaben umfangreiche Erneuerungen für 2020. Leider lassen aber nicht alle Vorhaben Gutes erwarten: Das kommende Jahr steht unter dem Stern des Globalbudgets.

Zunächst wird das Parlament das erste Kostendämpfungspaket behandeln. Diesem hat das Eidgenössische Departement des Innern (EDI) ein schlecht getarntes Globalbudget hinzugefügt: Neu sollen die Tarifpartner nach Artikel 47c des KVG «ungerechtfertigte» Mengen- und Kostenentwicklungen korrigieren [1] und dafür nach politischen Vorgaben eine "'akzeptable', d.h. gerechtfertigte Kostensteigerung» [2, S. $6133]$ definieren. Die Gesetzesvorlage sieht damit de facto die Festlegung eines Budgets vor, dessen Überschreitung Tarifreduktionen und Rückvergütungen nach sich zieht.

Ab Frühjahr 2020 wird dann mit der Vernehmlassung des zweiten Kostendämpfungspakets die vom Bundesrat angekündigte "Zielvorgabe für die Kostenentwicklung» [3] auf den Weg gebracht. Diese baut auf dem oben skizzierten Inhalt des ersten Kostendämpfungspakets auf, denn: «Die von den Tarifpartnern vereinbarten Massnahmen zur Steuerung der Kosten [...] würden dann dazu dienen, diese Zielvorgabe zu erreichen» [2, S. 6090].

Was in der Verwaltungssprache der Gesetzespakete unverdächtig klingt, bedeutet für unsere Gesundheitsversorgung ganz konkret: Der Versicherungsanspruch des Patienten endet zukünftig mit dem Budget. Denn Leistungen, die nicht oder unzureichend bezahlt oder sogar sanktioniert werden, wird kaum einer noch erbringen. Dies illustriert auch das Beispiel der Neurologin in Deutschland ab S. 196, die mittlerweile keine Patienten mehr annimmt [4]. Selbst die deutschen Mitglieder der bundesrätlichen Expertengruppe schreiben über die «Deckelung/Budgetierung der Gesamtvergütung» in Deutschland, die «kostendämpfende Wirkung auf Budgetebene [werde] insbesondere durch eine Reduktion der angebotenen Menge erreicht. Der Zugang kann durch die Budgetierung verschlechtert werden" [5, S. 8].
Es fallen also konkrete Leistungen an realen Patienten weg - welche Leistungen und bei welchen Patienten wird offengelassen. Wo bislang die Grundversicherung eine qualitativ hochwertige medizinische Versorgung im Krankheitsfall zusicherte, würde dieser Schutz zukünftig durch eine Ausgabenobergrenze eingeschränkt.

\section{Welche Leistungen bei welchen Patienten} wegfallen sollen, wird offengelassen.

Dass «Zielvorgaben» mit einem Versicherungsanspruch unvereinbar sind, wurde dem EDI jüngst in einem anderen Zusammenhang aufgezeigt. Unter dem Titel «Bund legt die Zahl der IV-Renten fest» [6] war zu lesen, das Bundesamt für Sozialversicherungen (BSV) lege für jede kantonale IV-Stelle Ziele in Bezug auf Renten und Kosten fest. Solche Zielvorgaben widersprächen jedoch einer unvoreingenommenen Prüfung von Rentengesuchen [7]: Die Chancen auf eine Rente dürfen nicht von den Zielvorgaben einer IV-Stelle abhängen. Diese Zielvorgaben sind darum nun Hauptgegenstand einer Untersuchung, die Bundesrat Berset «gegen die Aufsichtstätigkeit des BSV eingeleitet» hat [6, S. 9].

"Zielvorgaben" und Kostendeckel sind mit dem Versicherungsanspruch des Patienten unvereinbar.

Was heisst dies nun für "gerechtfertigte Kostensteigerungen" und "Zielvorgaben" in der Patientenversorgung? In der IV gilt berechtigterweise als stossend und rechtlich fragwürdig, dass politische «Zielvorgaben» den Versicherungsanspruch auf eine Rente untergraben können. Nur: Ist die Begrenzung des Anspruchs auf Leistungen der OKP-Grundversicherung durch einen politischen Kostenrahmen weniger fragwürdig? Aus Sicht der FMH dürfen die Chancen eines Patienten auf eine zeitnahe und qualitativ hochstehende Behandlung auch künftig nicht von Budgets und Zielvorgaben abhängen. Das Jahr der Erneuerung darf nicht das Ende des Versicherungsanspruchs einläuten. 